\title{
UN NUEVO MÉTODO DE EVALUACIÓN PARA SELECCIONAR UNA TECNOLOGÍA "APROPIADA" EN LA PRODUCCIÓN MASIVA DE VIVIENDAS
}

\author{
(A NEW EVALUATING METHOD TO SELECT AN “APPROPRIATE” TECHNOLOGY MASS \\ PRODUCTION HOUSING)
}

Mariana Enet, Arquitecto

Centro Experimental de la Vivienda Económica

Fecha de recepción:30-X-97

República Argentina

\section{RESUMEN}

Se trata de un método de evaluación predeterminado para tomar decisiones racionalmente en la selección de la tecnología más adecuada para la producción masiva de viviendas.

Permite, además, optimizar los puntos fuertes y prevenir los débiles en el proceso integral de fabricación, transporte, montaje, calidad resultante y mantenimiento final en relación a las necesidades de los distintos sectores que la utilizarán: usuarios, fabricantes, transportistas, constructores y vendedores inmobiliarios. También es una herramienta indispensable cuando se desea transferir una innovación tecnológica al medio productivo.

La utilización de métodos sistemáticos en la selección de tecnologias mejora la calidad en los alojamientos y produce óptimos rendimientos económicos.

\section{SUMMARY}

A method is described for predetermined evaluation that will help making rational decisions on the selection of the most acurate technology for mass production housing.

It allows an optimization of strong points and the prevention of the weak ones within the whole process -manufacture, transport, fitting, quality control and maintenance-, in accordance with the needs of the different sectors fabricators, transporters, builders, real estate agents and dwellers. It is also an essential tool for transferring the technological innovation onto the actual production process.

The use of systematic methods for the selection of the appropriate technology results in quality improvement and economical savings.

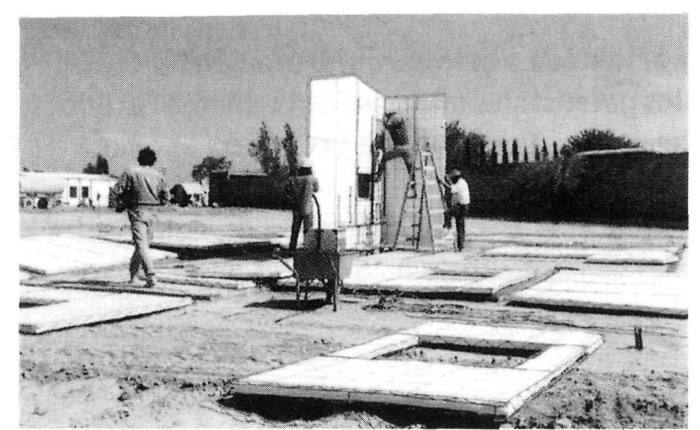

Foto 1

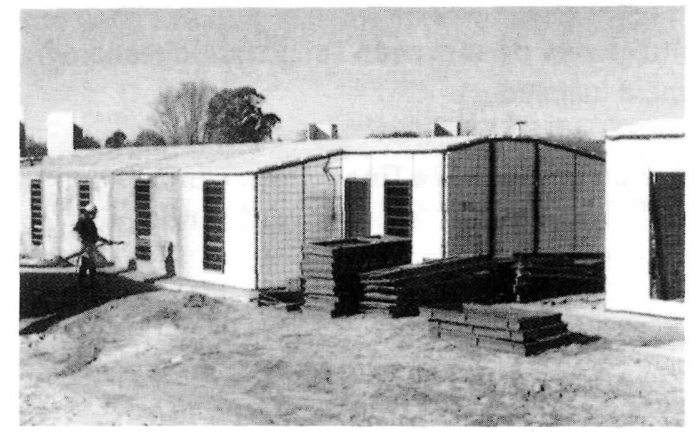

Foto 2

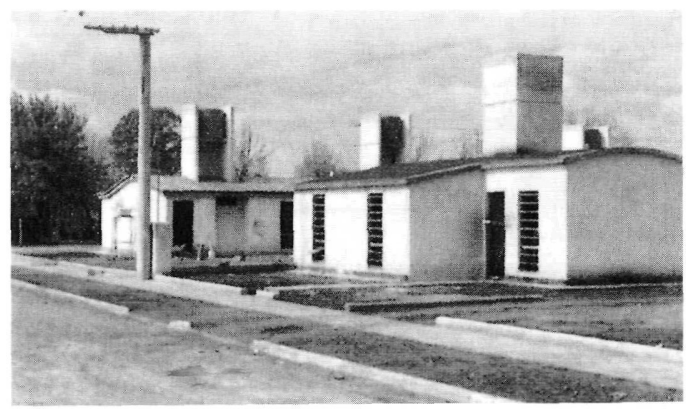

Foto 3 


\section{INTRODUCCIÓN}

Cuando se va a realizar una producción masiva de viviendas se ponen en juego amplios recursos económicos, humanos y organizativos pero ¿cuántas veces nos detenemos a realizar estudios de factibilidad y eficiencia para utilizar otras tecnologías además de las tradicionalesracionalizadas?

Un estudio de licitaciones de planes habitacionales realizado por el IPV (Instituto Provincial de la Vivienda de Córdoba) en el año 1994, arrojó el resultado de que en 10 años el $99 \%$ de las empresas constructoras utilizaba el conocido sistema tradicional-racionalizado de bloques y viguetas de hormigón, mientras que en sólo el $1 \%$, representado por dos empresas constructoras, se habian utilizado sistemas prefabricados ${ }^{1}$.

Este dato relevante nos llevó a preguntarnos cuáles eran las causas ¿una evidente superioridad técnica, económica, financiera, legal, de calidad? ¿o era desconocimiento de otras alternativas tecnológicas? ¿es racional y lógico establecer estas prohibiciones o. por el contrario, desarrollar mecanismos adecuados para un eficiente control de calidad, tanto de los sistemas prefabricados como de los tradicionales?

Para contestarnos esta pregunta utilizamos un conjunto de métodos que permitió analizar la situación desde un punto de vista contextual y sistemático.

. Evaluaciones cualitativas a los usuarios (productores, constructores y familias).

- Evaluaciones de mercado (analizando tecnologías de referencia instaladas).

. Evaluación económica financiera, comparativa con la tecnología tradicional racionalizada, utilizada en las licitaciones.
. Un método de evaluación técnico-funcional comparativa, que es la que se va a desarrollar en este artículo ${ }^{2}$.

Conceptos en los que se basa

\section{. Concepción de tecnología}

Se basa en la concepción de que la tecnología no es solamente un hecho constructivo sino que, por el contrario, es válida en función de la adecuación de su respuesta a un medio dado y en relación a la respuesta técnica funcional que se espera de ella.

Esta es la línea de tecnología "adecuada" como la define Tudela: "La tecnología adecuada es un término que implica una visión particular de la sociedad y de la tecnología, sugiere que esta última no es neutral ni evoluciona en una dirección única. Reconoce que los distintos grupos culturales y geográficos tienen tecnologías diversas que son apropiadas a sus circunstancias y que la autodeterminación tecnológica es esencial para la identidad cultural y la independencia política" ${ }^{3}$.

\section{. Prevención - optimización}

Es un conjunto de métodos y herramientas que permitirán prevenir las patologías desde el inicio de la producción, diseño, montaje y mantenimiento.

Se obtendrán datos clave para una adecuada planificación integral de obra que permitirá una optimización en el uso de los recursos y evitará gastos y actividades no previstas en todo el proceso de producción de obra ${ }^{4}$.

. Está orientada a brindar información técnica-funcional a los potenciales usuarios de la misma (productores, constructores, familias, etc.) (Gráfico 1).

Este enfoque se basa en revisar la tecnología desde los intereses de los distintos actores que pueden hacer uso de

Gráfico 1

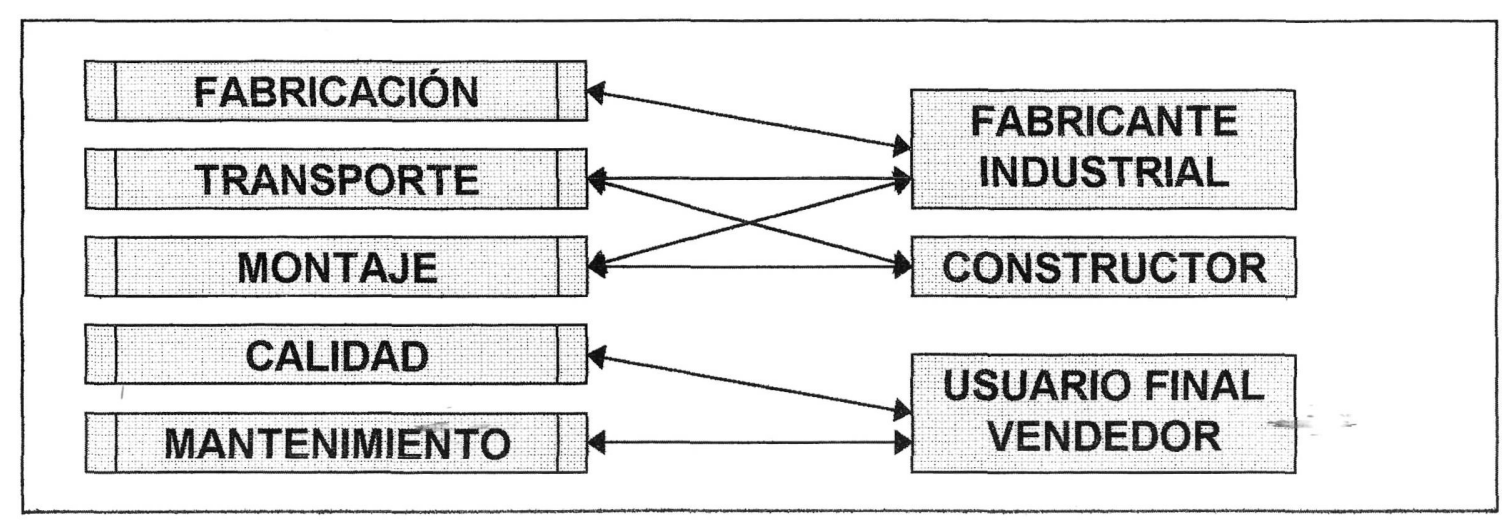


ella, con el objetivo de rediseñar o adaptar aspectos clave que se descubren al transferirse al medio concreto.

\section{OBJETIVO}

Conocer la capacidad de inserción de una innovación tecnológica en el medio productivo, describiendo sus potencialidades y debilidades en las distintas etapas de transferencia.

Descubrir la alternativa tecnológica más apta para la producción masiva de viviendas.

\section{Objetivos particulares}

- Verificar, ajustar o reformular la producción a escala masiva.

- Verificar la capacidad de competencia del sistema constructivo prefabricado, seleccionado con la tecnología constructiva posicionada en el medio para la producción masiva.

- Verificar la capacidad de transferencia de la innovación en los distintos momentos de fabricación, transporte, montaje, calidad final obtenida y mantenimiento.

- Verificar la adecuación del producto a las necesidades de los distintos usuarios del mismo (productores, constructores, comerciantes, familiares, etc.).

\section{PASOS DE LA EVALUACIÓN}

1) Determinar, por estudio de mercado, la tecnología de "referencia" que se encuentra posicionada parael segmento o sector al cual nos dirigimos.
2) Realizar un diseño equivalente entre el producto de "referencia" y la tecnología prefabricada que queremos evaluar.

3) Realizar una descripción y análisis detallado de los dos sistemas (si es posible, tomar datos de la obra directa).

4) Introducir los datos en las 11 fichas comparativas de ambas tecnologías.

5) Procesar los datos y calcular el nivel de eficiencia obtenida por indicador, variable, etapa y, finalmente, el sistema.

6) Analizar y representar, comparativamente, las dos tecnologías. Realizar cartilla comparativa.

\section{PAUTAS METODOLÓGICAS}

¿Qué se evalúa?

Nivel de eficiencia relativo para la producción masiva y calidad final obtenida.

¿Cuál es la escala de análisis?

Este método pretende incorporar nuevos indicadores y categorías técnico-funcionales que permitan describir las ventajas relativas para la producción masiva y calidad final obtenida. Observando al objeto a través de las distintas etapas de la producción y en función de los usuarios que pueden utilizarla.

Los resultados serán expuestos, en primer lugar, observando al objeto en general, analizando su aptitud global para todas las etapas y todos los usuarios (análisis verde).

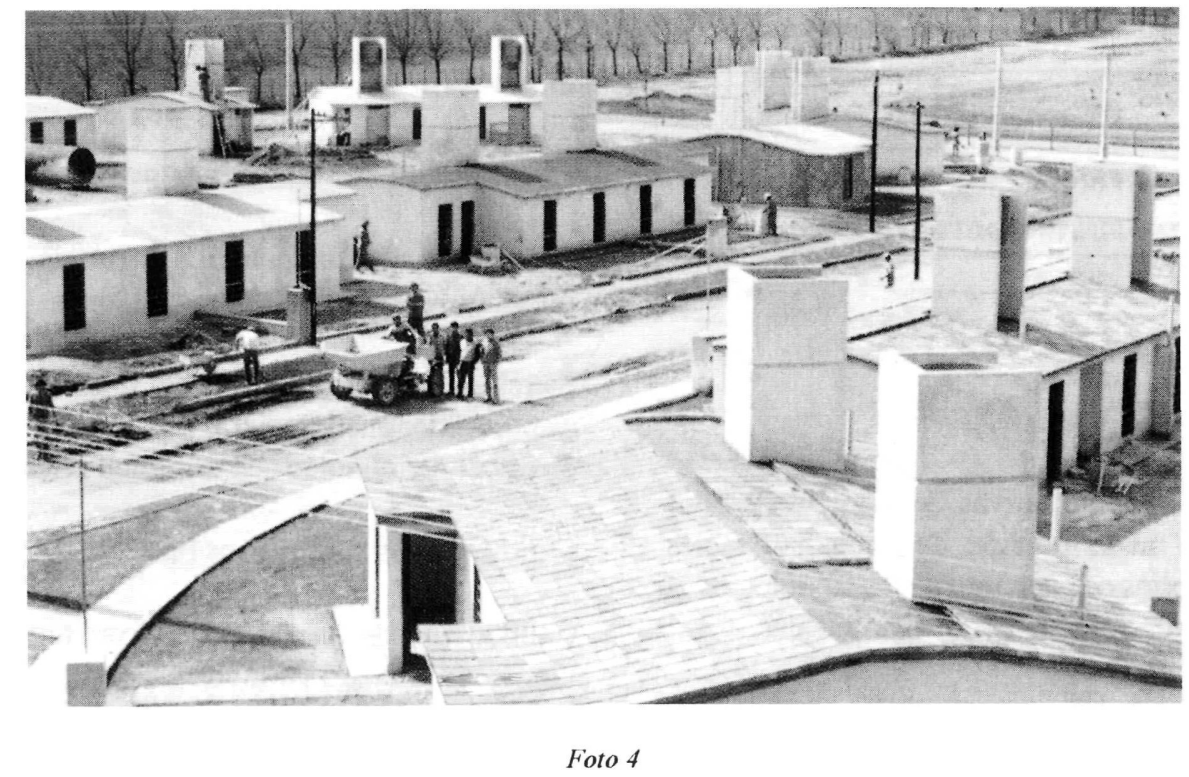


En segundo término, en función del tipo de usuario al que le queremos trasferir o el tipo de uso que quiere hacer del sistema, podemos acceder a una evaluación más exhaustiva del mismo por etapas (análisis marcado en amarillo) (Gráfico 2).
Estas fichas no son fijas: el evaluador puede utilizar todas o un grupo de ellas en función de la profundidad del análisis o flexibilización de la medición de las categorías en función de los datos que posee sobre el objeto 5 .

Gráfico 2

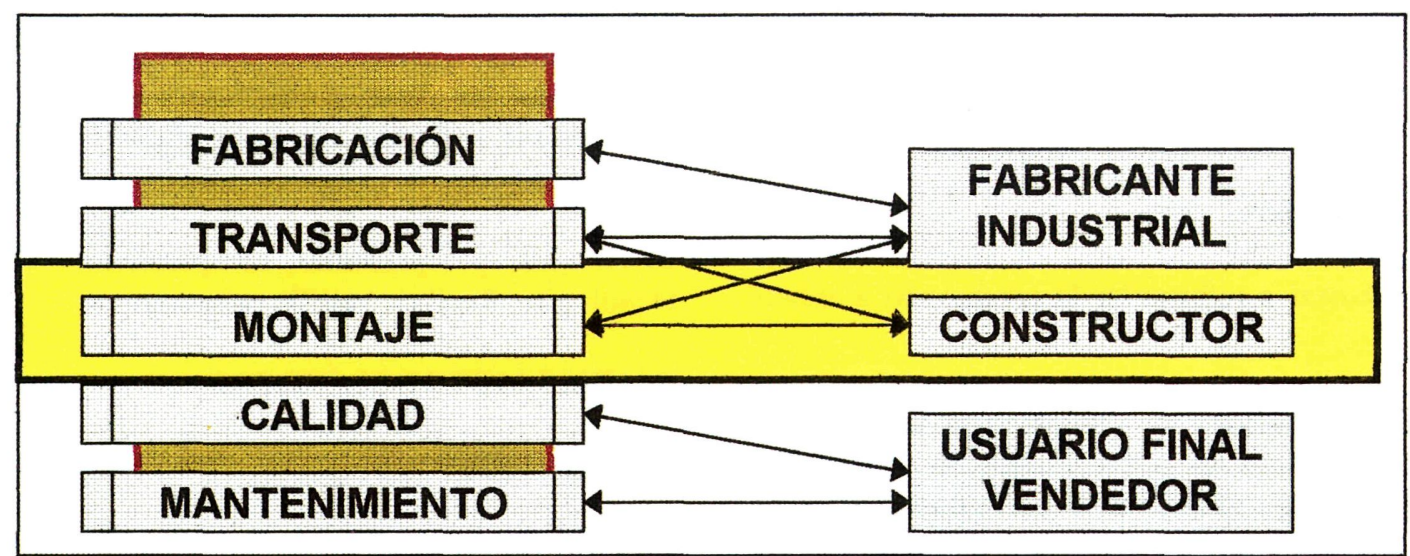

\section{PARÁMETROS DE VALORACIÓN}

El parámetro comparativo será el nivel de ejecución técnica en relación al parámetro considerado como ideal.

El análisis técnico-funcional se basará en un análisis cuantitativo con el objetivo de demostrar a terceros su validez.

El método debe complementarse con otros análisis más blandos como encuesta a usuarios, productores y constructores.

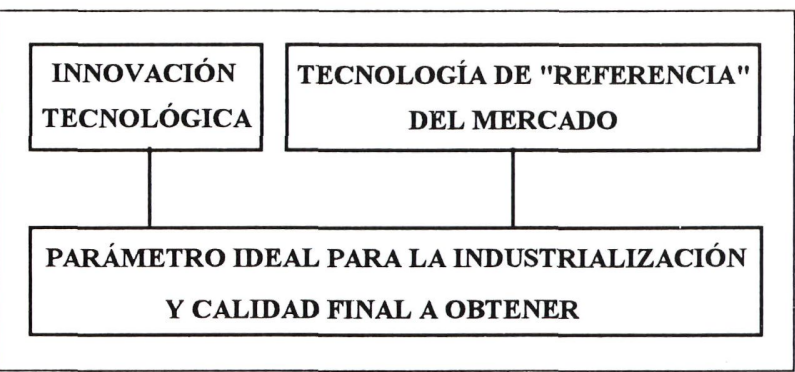

\section{TÉCNICAS DE EVALUACIÓN}

Se utilizarán técnicas cuantitativas de medición prefijadas consistentes en la valoración de temas, variables, indicadores y categorías de cada una de las tecnologías en función de parámetros considerados como ideales.

Para ello se ha diseñado un conjunto de 11 fichas en las cuales se mide el nivel eficiencia de cada tecnología.

(c) Consejo Superior de Investigaciones Científicas Licencia Creative Commons 3.0 España (by-nc)
- Temas variables e indicadores

A modo de ejemplo citaremos la forma de construcción de algunas de las variantes e indicadores que van caracterizando la aptitud de la tecnología para la producción masiva.

Variable facilidad en la obtención de suministros.

Supuesto óptimo para la producción masiva en la obtención de suministros.

La facilidad de obtención de suministros es directamente proporcional a la posibilidad de seleccionar proveedores, obtener una calidad estable, una oferta masiva, continua y de producción local.

- Se establecen los indicadores y las categorías en base al supuesto

\section{- Posibilidad de seleccionar proveedores}

Provisión no monopolística / provisión monopolística restringida / provisión monopolística.

Se refiere a que la dependencia sobre un determinado tipo de proveedor puede generar inconvenientes en el costo y en otros condicionantes que un único pueda imponer.

Calidad de los suministros básicos:

Calidad invariable / Calidad con algunas alteraciones menores / Calidad variable.

La variedad se establece en peso, volumen, textura, resistencia, color, etc. 
Se refiere a que la variación en las condiciones del suministro básico puede atentar contra la calidad prevista en el sistema.

Continuidad de la oferta:

Oferta permanente / Oferta estacional o intermitente / Oferta esporádica

Se refiere a la posibilidad de desarrollar la tecnología con continuidad y no depender de períodos estacionales o productores esporádicos de suministros básicos.

Amplitud de la oferta:

Escala masiva / Escala media / Escala minorista

Se refiere a la posibilidad de conseguir los suministros básicos en la escala que se necesita, sin necesidad de recurrir a muchos de ellos cuando se necesita un volumen importante de suministros.

Origen del suministro:

Suministro fabricado localmente / suministro importado reemplazable / suministro importado no reemplazable.
Se refiere a la posibilidad de conseguir suministros sin depender de las leyes de la importación o a la posibilidad que tienen los mismos de reemplazarse.

\section{FICHA DE PROCESAMIENTO Y CÁLCULO DE LA INFORMACIÓN}

A modo de ejemplo podemos mostrar una de las fichas de procesamiento y cálculo con sus fórmulas (Tabla I).

Ésta es comparable con una idéntica para colocar los valores de la tecnología tradicional racionalizada.

\section{DISEÑO GENERAL DE LA EVALUACIÓN}

Estos resultados se obtienen del procesamiento y cálculo de las 11 fichas, una para la tecnología prefabricada y otra para la tecnología tradicional racionalizada. Se puede aplicar con distintos niveles de profundidad, según sea la necesidad de evaluación (Tabla II).

Ésta, es una tabla síntesis que va complementada con información cuantitativa y cualitativa específica.

Para una información más profunda se busca en las fichas de cada etapa, pudiendo analizarse de forma especifica.

\begin{tabular}{|c|c|c|c|c|c|c|c|c|c|}
\hline \multicolumn{10}{|c|}{ FICHA 7 A- ETAPA MONTAJE- SISTEMA FC2 } \\
\hline \multicolumn{10}{|c|}{ Variable: Facilidad de control } \\
\hline & \multicolumn{3}{|c|}{ Racionalización del control } & \multicolumn{3}{|c|}{ Piezas reducidas } & \multicolumn{3}{|c|}{ Control previo } \\
\hline & $\begin{array}{c}>60 \% \text { de } \\
\text { partidas con } \\
\text { racionaliz. } \\
\text { en el control }\end{array}$ & $\begin{array}{c}30 \% \text { y } 60 \%] \\
\text { de partidas } \\
\text { con } \\
\text { racionaliz. } \\
\text { en el control } \\
\end{array}$ & \begin{tabular}{|c|}
$<$ del $30 \%$ \\
de partidas \\
con \\
racionaliz. \\
en el control \\
\end{tabular} & $\begin{array}{l}>\text { del } 50 \% \\
\text { de partidas } \\
\text { con piezas } \\
\text { reducidas }\end{array}$ & $\begin{array}{c}\{20 \% \text { y } 50 \%] \\
\text { de partidas } \\
\text { con piezas } \\
\text { reducidas }\end{array}$ & $\begin{array}{c}<\text { del } 20 \% \text { de } \\
\text { partidas con } \\
\text { piezas } \\
\text { reducidas }\end{array}$ & $\begin{array}{c}>\text { del } 20 \% \\
\text { de partidas } \\
\text { con control } \\
\text { previo }\end{array}$ & \begin{tabular}{|c|}
$20 \%$ y \\
$10 \%]$ de \\
partidas con \\
control \\
previo \\
\end{tabular} & $\begin{array}{c}<\text { del } 10 \% \\
\text { de partidas } \\
\text { con control } \\
\text { previo }\end{array}$ \\
\hline $\begin{array}{c}\text { Calificación del } \\
\text { indicador }\end{array}$ & \multicolumn{3}{|c|}{100} & \multicolumn{3}{|c|}{100} & \multicolumn{3}{|c|}{50} \\
\hline $\begin{array}{c}\text { Ponderación del } \\
\text { indicador }\end{array}$ & \multicolumn{3}{|c|}{10} & \multicolumn{3}{|c|}{2,5} & \multicolumn{3}{|c|}{2,5} \\
\hline & \multicolumn{3}{|c|}{1000} & \multicolumn{3}{|c|}{250} & \multicolumn{3}{|c|}{125} \\
\hline & \multicolumn{9}{|c|}{$1375 / 15$} \\
\hline $\begin{array}{l}\% \text { de eficiencia } \\
\text { de la variable }\end{array}$ & \multicolumn{9}{|c|}{91,66} \\
\hline
\end{tabular}

$\mathrm{Ix}=1 \times \mathrm{Ca}+1 / 2 \times \mathrm{Cb}+0 \times \mathrm{Cc}$

$$
\begin{array}{ccc}
C a=1 \times 100+1 / 2 \times 0+0 \times 0 & C b=1 \times 100+1 / 2 \times 0+0 \times 0 & C c=1 \times 0+1 / 2 \times 100+0 \times 0 \\
C a=100 & C b=100 & C c=50
\end{array}
$$

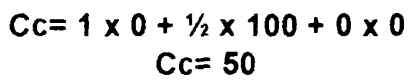
$C$ variable $=(\mathrm{Ca} \times \mathrm{Pa}+\mathrm{Cb} \times \mathrm{Pb}+\mathrm{Cc} \times \mathrm{Pc}) / \mathrm{P}$ variable ( $\mathrm{P}$ es la ponderación del indicador $\mathrm{y}$ de la variable)

$C v=(100 \times 10+100 \times 2,5+50 \times 2,5) / 15 \quad C_{v}=(1000+250+125) / 15 \quad C v_{v}=1375 / 15 C_{v}=91,66$ 


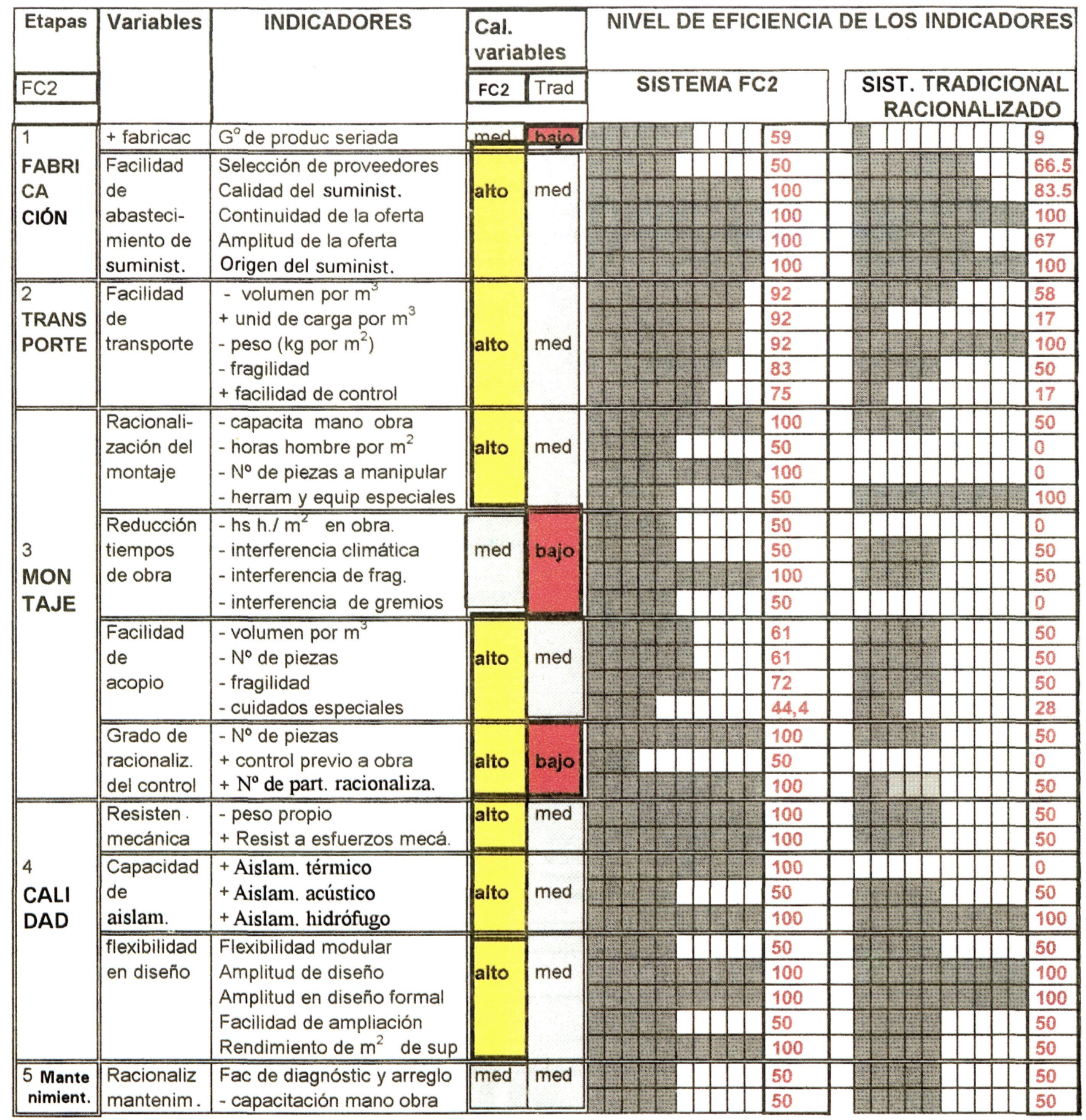

\section{RESULTADOS GENERALES DE APTITUD EN EL ASPECTO TÉCNICO-FUNCIONAL}

Este resultado es producto de considerar su aptitud para la producción masiva en la etapa de fabricación, transporte, montaje, calidad y mantenimiento (Tabla III).

El sistema $\mathrm{FC} 2$ es un $24 \%$ más apto para la producción masiva que el sistema tradicional racionalizado de bloques y viguetas de hormigón en el aspecto técnico-funcional (Gráfico 3).

(c) Consejo Superior de Investigaciones Científicas Licencia Creative Commons 3.0 España (by-nc)
. Resultados generales de aptitud en las grandes etapas
de producción y uso de los sistemas.

Estos resultados son el producto de considerar, para cada etapa, las distintas variables que intervienen y cuál es su nivel de aptitud para la producción masiva (Tabla IV).

Se observa una ventaja comparativa del Sistema FC2 sobre el sistematradicional en la producción masiva de viviendas, especialmente en la posibilidad de producción seriada y fabricación en taller, en la aptitud relativa para el transpor- 


\begin{tabular}{|l|c|}
\hline \multicolumn{1}{|c|}{ SISTEMA CONSTRUCTIVO } & PORCENTAJE DE APTITUD \\
\hline SISTEMA FC2 ${ }^{*}$ en altura & $70 \%$ \\
\hline SISTEMA TRADICIONAL RACIONALIZADO & $46 \%$ \\
\hline
\end{tabular}

Gráfico 3

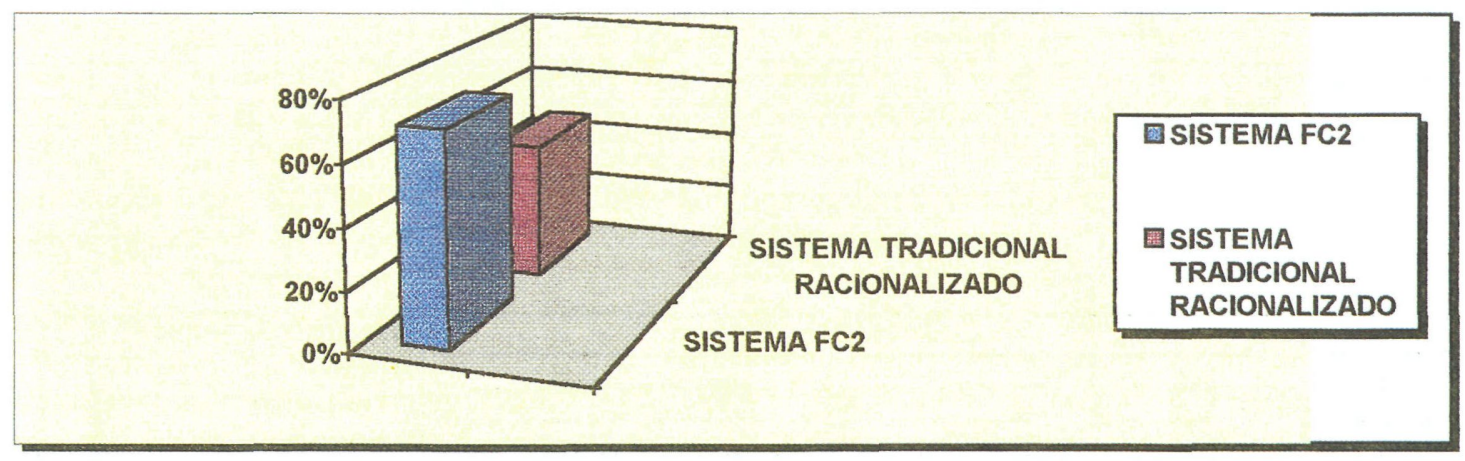

TABLA IV

SISTEMA FC2 SISTEMA TRADICIONAL

\begin{tabular}{|l|l|l|l|l|l|l|l|l|l|l|l|l|l|l|l|}
\hline ETAPAS & \multicolumn{3}{|c|}{ GRADO DE APTITUD } & & \multicolumn{6}{|c|}{ GRADO DE APTITUD } & RELACIÓN \\
\hline FABRICACIÓN & & & & & & & 74,5 & & & & & & 46 & 28,5 \\
\hline TRANSPORTE & & & & & & & 87 & & & & & & 48 & 39 \\
\hline MONTAJE & & & & & & & 67 & & & & & & 28 & 39 \\
\hline CALIDAD & & & & & & & 71 & & & & & & 56 & 15 \\
\hline MANTENIMIENTO & & & & & & & 50 & & & & & & 50 & 0 \\
\hline
\end{tabular}

te de componentes y suministros y en aptitud de montaje de viviendas in situ.

En cuanto a calidad, se observa una ventaja relativa sobre el tradicional, no así en el mantenimiento, donde los dos sistemas presentan una aptitud similar (Gráfico 4).

. Resultados generales de aptitud en las variables de producción y uso de los sistemas (Tabla V) (Gráfico 5).

\section{CONCLUSIONES}

Estos métodos son herramientas indispensables para clarificar las ventajas comparativas de la tecnología en la producción masiva, así como en las posibilidades de programación y previsión para la etapa de producción.

Permite obtener datos globales del comportamiento y profundizar en aspectos específicos en cada una de las etapas y en función de la visión de cada uno de los usuarios.

Gráfico 4

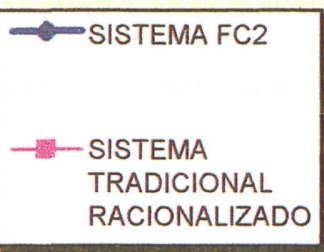


SISTEMA FC2 SISTEMA TRADICIONAL

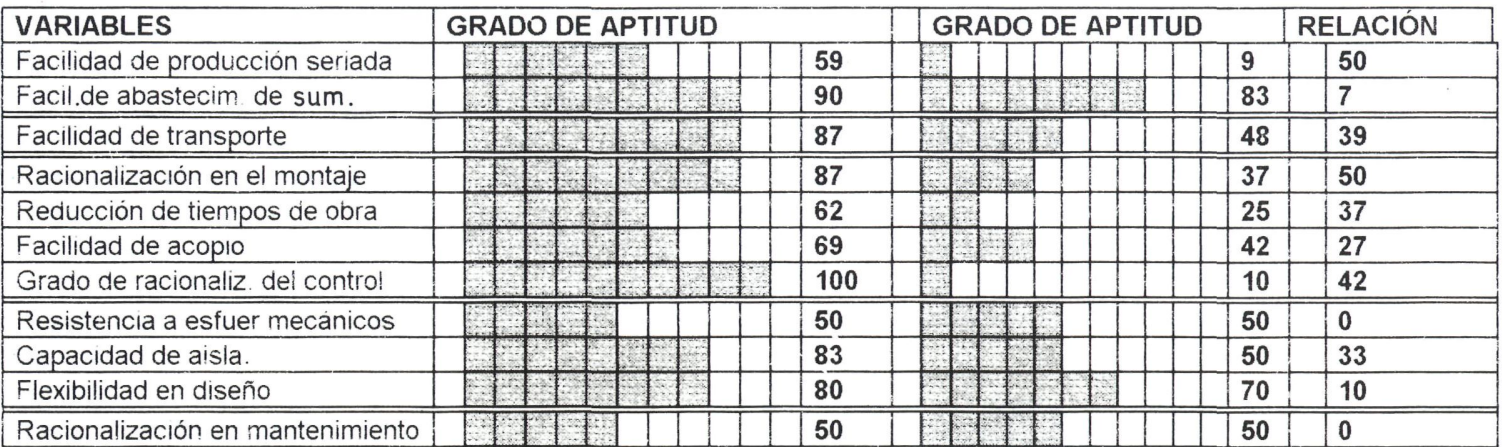

Gráfico 5

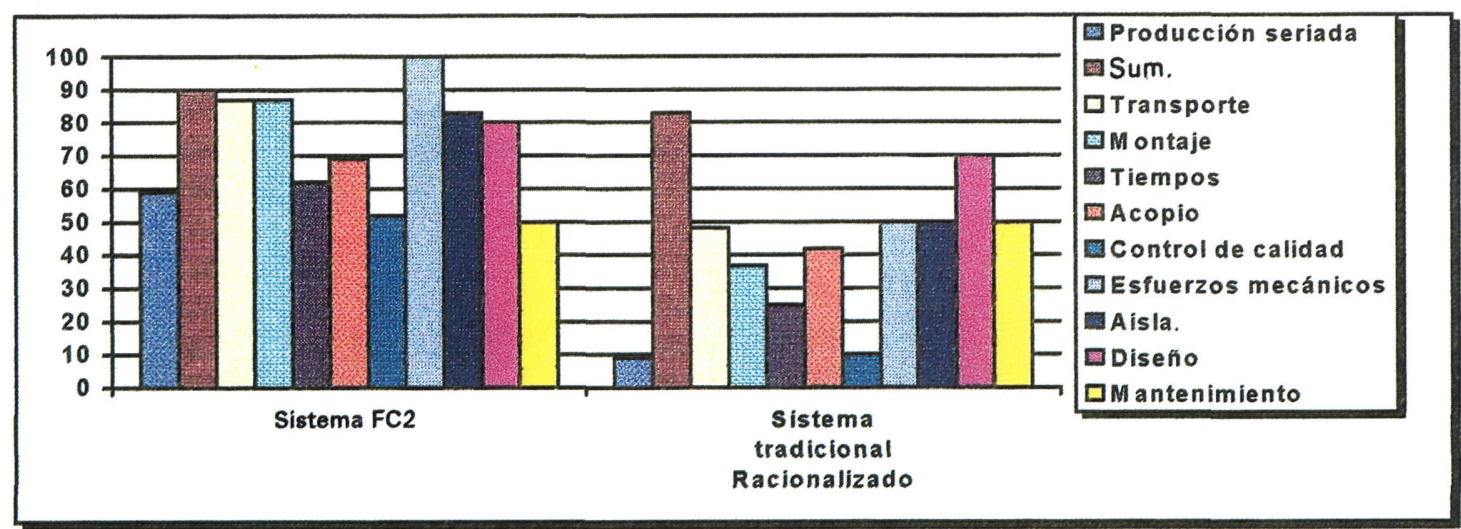

La utilización de este método por parte de instituciones gubernamentales, empresarios o investigadores de la construcción comoinstrumento de selección de la alternativa más apta o de la tecnología que garantice el cumplimiento de condiciones básicas de calidad, permite prever patologías o desajustes en su aplicación y la optimización en la utilización de los recursos.

En síntesis, el uso racional de herramientas que permitan seleccionar alternativas tecnológicas en vez de desecharlas, por desconocimiento o prejuicios, puede ser el camino que permita tecnificar la construcción, avanzar en la eficiencia de producción y en una óptima calidad final.

\section{BIBLIOGRAFÍA}

- MONJO CARRIÓ, Juan, 1986. "Propuesta de evaluación de sistemas constructivos". Informes de la Construcción. Vol. 38. N $N^{\circ} 385$, págs 5-29.

- TUDELA, Fernando, 1987. "Tecnología para los asentamientos humanos. Guía metodológica para la selección"

- ENET, Mariana, 1996. "Método de evaluáción de innovaciones tecnológicas en la etapa de transferencia al medio". Publicación CEVE.

(c) Consejo Superior de Investigaciones Científicas Licencia Creative Commons 3.0 España (by-nc)

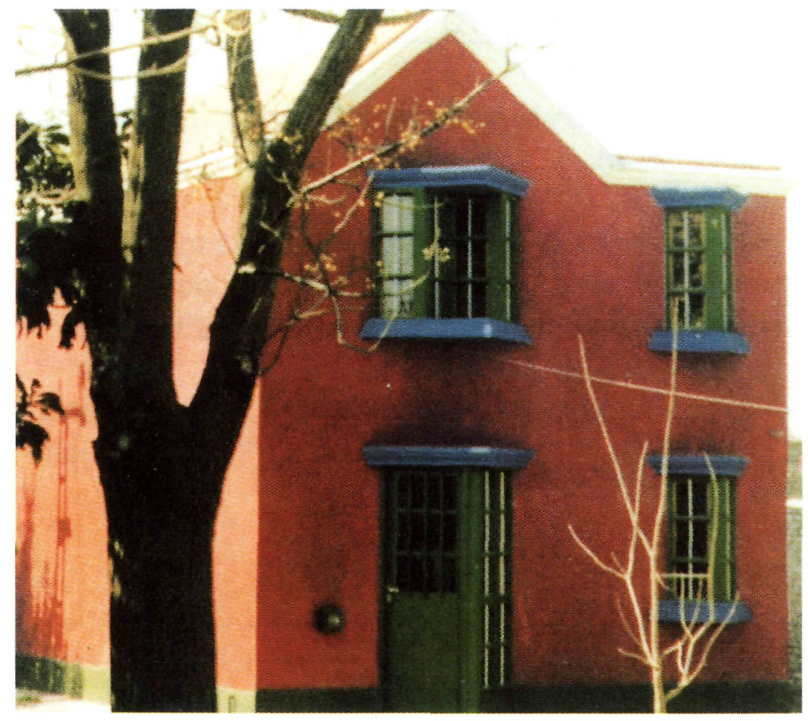

Foto 5

\section{NOTAS}

1 "Entre mayo de 1988 y abril de 1989, la Secretaría de Vivienda y Calidad Ambiental, a través de la Dirección de Tecnología, realizó una evaluación sobre los conjuntos habitacionales construidos con sistemas no tradicionales de producción industrial, determinando patologías que afectaban a la durabilidad, habitabilidad y seguridad de los mismos. En mayo de 1989, el 
Instituto Provincial de la Vivienda (IPV) de la provincia de Buenos Aires prohibe el uso de sistemas constructivos no tradicionale sindustrializados en el ámbito de dicha provincia, haciéndose luego la prohibición extensiva a otras provincias".

"Auditoría tecnológica en paneles de base cerámica" Gastron y otros. Revista AREA, agosto 1996.

${ }^{2}$ A modo de síntesis, podemos destacar algunas de las causas que detectamos, además del desconocimiento y temor a utilizar una tecnología que no le es habitual.

a) Resistencia de los usuarios finales a los sistemas prefabricados que se asocian a vivienda económica de bajo status y de baja calidad.

b) Resistencia de los organismos estatales para declararlos técnicamente "aptos".

c) Inadecuación o imprevisión de un sistema de certificación de obra que contemple la producción de componentes en fábrica. Esto implica prever un análisis financiero específico para el movimiento del dinero.

d) Tareas suplementarias para realizar una "adaptación tecnológica" a los recursos humanos, materiales, climáticos y normativos del área donde se piensa aplicar por primera vez.
3 Fernando Tudela "Tecnologías para los asentamientos humanos".

${ }^{4}$ Este método coincide con las recomendaciones del Primer Congreso Latinoamericano de patologías de la Construcción realizado en abril de 1991 en Córdoba, cuyas conclusiones recomiendan profundizar en la "prevención" de patologías

${ }^{5}$ Algunas de estas categorías están sujetas a prueba, ya que no han sido utilizadas en otros análisis que nos puedan confirmar la certeza $v$ racionalidad de los estándares exigidos. Por ejemplo, en el indicador "Disminución del control en obra" la categoría más alta es $>$ de $20 \%$ de partidas con control previo a obra: la categoría media es entre [ $20 \%$ y $10 \%]$ de partidas con control previo a obra; la categoría más baja $<$ de $10 \%$ de partidas con control previo a obra.

Podemos tener un margen de duda al seleccionar cómo dividiremos las partidas y otro en función de en qué medida es normal (para un número considerable de sistemas prefabricados el control previo a obra) los estándares prefijados.

Lo que se considera un avance es comenzar a indagar sobre estos aspectos, que son determinantes cuando se realiza un plan masivo de viviendas en función de la calidad final obtenida, la reducción de tiempos de obra y la clasificación técnica del control. Éste podrá verificarse cuando este método sea probado en un número considerable de casos.

\section{Publicación del Instituto Eduardo Torroja - CSIC}

\section{Número monográfico de INFORMES}

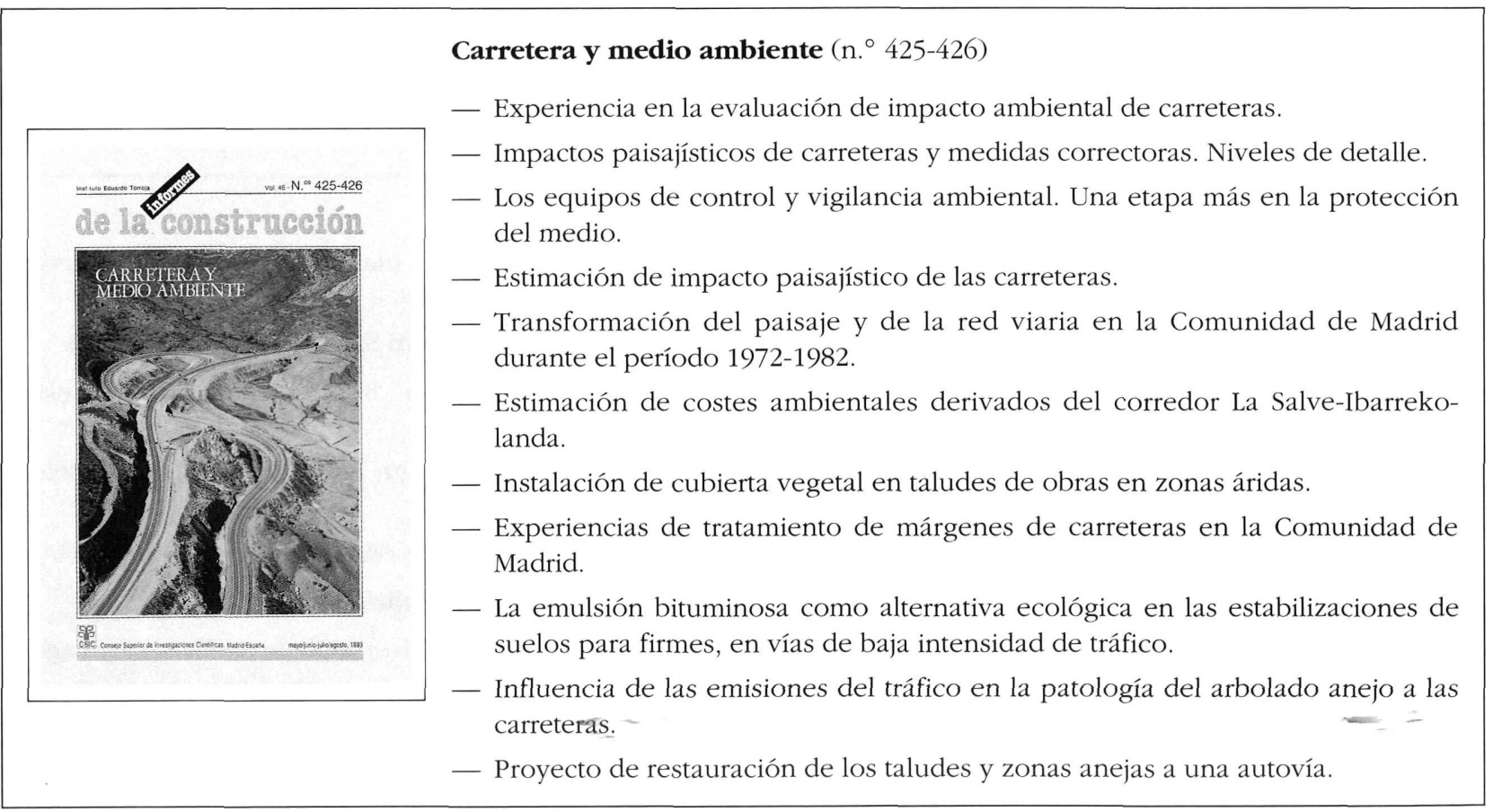

